\title{
The effect of a response-contingent stimulus introduced into a fixed-interval schedule at varying temporal placement'
}

J. FARMER AND W. N. SCHOENFELD

QUEENS COLLEGE OF THE CITY UNIVERSITY OF NEW YORK

A response-contingent brief light $\left(S_{1}\right)$ is made to occur at systematically varied temporal positions in a $60 \mathrm{sec}$. fixedinterval reinforcement schedule. Functions are presented showing the effects of the $S_{1}$ at all points in the fixedinterval. A comparison with previously reported data show that these effects do not depend on the presence of a response contingency requirement for $S_{1}$ occurrence.

In two experiments reported elsewhere (Farmer \& Schoenfeld, 1966) we defined an irreducibly primitive paradigm for exploring stimulus effects on steady-state responding. This basic paradigm was illustrated by the non-response contingent introduction of a brief (6-sec.) light (notated $S_{1}$ ) into each interval of a $60 \mathrm{sec}$. fixedinterval (FI) reinforcement schedule. In those experiments, the independent variable was the temporal position of $\mathrm{S}_{1}$ in each interval of $60 \mathrm{sec}$. FI schedule. Clearly visible behavioral effects were found that centered on $S_{1}$ and that depended closely on $S_{1}$ 's temporal position in the interval. The present study shows that these findings remain relatively unchanged when the occurrence of $S_{1}$ is made response contingent. Method

Subjects. Two White Carneaux hen pigeons (No. 261 and No, 268), 6-8 yr. old, were maintained at $80 \%$ ad libitum body weight throughout the experiment.

Apparatus. The experimental chamber was a Lehigh Valley Electronics pigeon chamber Model No. 1519C, containing a pecking key as operandum. A minimum of $25 \mathrm{~g}$ on this key was required for microswitch closure. Transistorized digital logic components programmed stimulus changes and reinforcement $\left(S^{R}\right)$ delivery. Data were recorded on Sodeco counters.

Procedure. After shaping the response (with $\mathrm{S}^{\mathrm{R}}$ being 3 sec. access to mixed grain) to the key transilluminated by $1.8 \mathrm{log} \mathrm{ft}$. lamberts white diffused light, the two pigeons were exposed over 40 daily sessions, each of which terminated after $61 \mathrm{~S}^{R_{S}}$, to the FI $60 \mathrm{sec}$. reinforcement schedule. The intervals of this schedule were timed from the end of the preceding $S^{R}$. In the next experimental phase, beginning on day 41 , a key-peck changed the transillumination of the key from white to green light $\left(\mathrm{S}_{1}\right)$ of $0.1 \mathrm{log} \mathrm{ft}$. lambert intensity for $6 \mathrm{sec}$. out of each 60 sec. FI cycle, the key continuing to be white for the remaining 54 sec. of each interval. The temporal position within each $60-\mathrm{sec}$. interval at which the response was allowed to produce the $6 \mathrm{sec}$. green light was systematically varied, with training given at each temporal position for seven days (seven 1-hr. daily sessions). For the first seven days of the experimental phase, the response-contingent $s_{1}$ occurred during the $6 \mathrm{sec}$. from the 48 th to the 54 th sec., inclusive, in the FI cycle. Six sec. after the change from green back to white, the $60 \mathrm{sec}$. interval timed out and $\mathrm{s}^{\mathbf{R}}$ occurred with the next response. If no responses occurred at 48 sec., the key remained white and the timing out of the remaining portion of the $60-\mathrm{sec}$. cycle did not take place until a response initiated the 6-sec. green light period, after which timing out occurred as before. This means, then, that intervals would exceed $60 \mathrm{sec}$. by the amount of delay between the time when a response could produce the green light and when that response actually occurred. This type of schedule has been called "chain FIFI"' (Ferster \& Skinner, 1957), although more usually the latter schedule has employed a continuing stimulus in each component as contrasted with our present use of only a 6-sec. cue for the second component. The difference between the present form of "chain FIFI" and what might be labelled "multiple EXTFI" is the absence of response contingency in the latter. (If we consider $S_{1}$ itself as a stimulus defining a separable schedule component, then comparisons are possible between our procedure and 3-ply MULT and chain schedules.) Five temporal assignments of $S_{1}$ were used in the experiment, with the birds exposed to each for seven days. These placements, specified as time since the preceding $S^{R}$, and the order in which they were used, were 48-53.99 sec.; 36-41.99 sec.; 24-29.99 sec.; 12-17.99 sec.; 0-5.99 sec. The data reported here are mean performances for the last five days (days 36-40) of the 40-day exposure to FI $60 \mathrm{sec}$,, and for the last two of each block of seven days at each of the five temporal assignments of (response-contingent) $S_{1}$.

\section{Results and Discussion}

Figure 1 plots response rates as the dependent variable, and shows in the top two panels baseline FI patterns of responding that emerged after 40 days of training and before the introduction of $s_{1}$. These patterns are the typically reported ones of low response rates following $S^{R}$ which gradually increase and become maximal just prior to the next $S^{R}$ delivery. Bird 261 maintained a high rate of responding in $S_{1}$ when $S_{1}$ onset was at the shortest (12 sec.) temporal separation from the next available $\mathbf{S R}$. With increasing temporal separation between $S_{1}$ and the next available $S^{R}$ (i.e., the $S_{1}-S^{R}$ interval), rate in $S_{1}$ falls to low values and scalloping emerges before and after its occurrence. At yet longer $S_{1}-S^{R}$ intervals (48 sec.) scalloping 


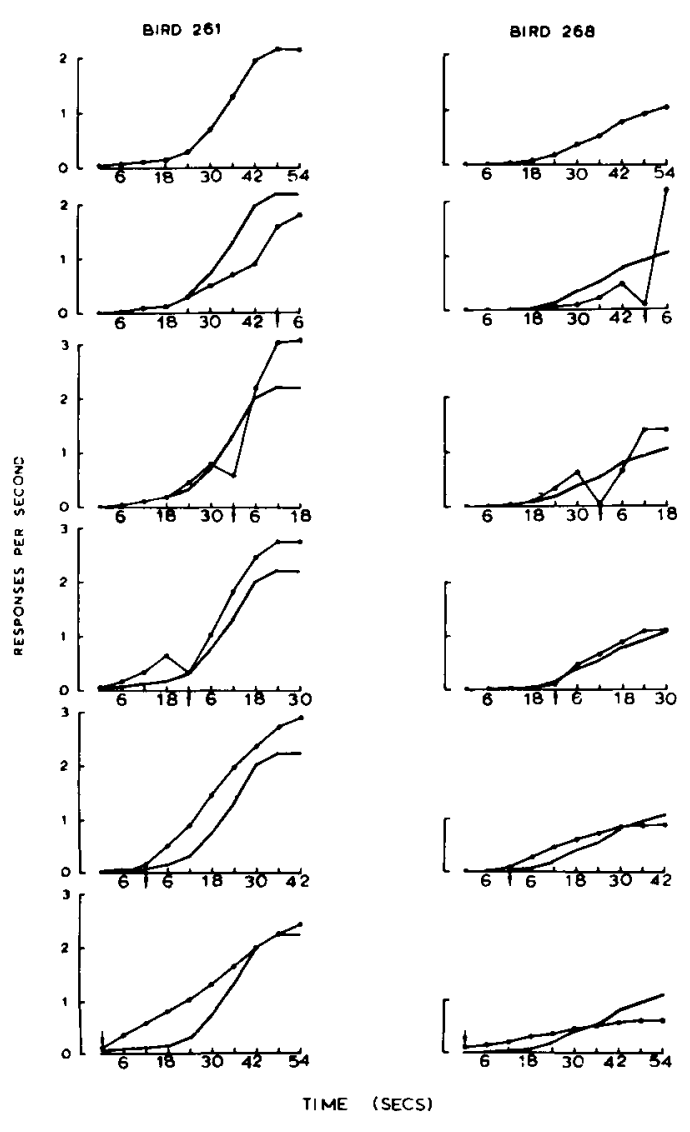

Fig. 1. Performances of Birds 261 and 268. Arrows indicate the response-contingent occurrence of $s_{1}$ onset. The top panels in each column (without arrow) are baseline performances on the 60 sec. FI schedule before the introduction of $s_{1}$; each datum point here represents the response rate for each successive 6-sec. in the FI interval averaged across 300 intervals (the last 5 sessions taken as criterion performances from 40 approximately 1-hr daily sessions of training). This baseline function is retraced in all panels for comparison purposes. Data points in all other panels are averages of 120 intervals (the last 2 sessions from 7 approximately 1-hr daily sessions taken as criterion performances). The numbers on the abscissae mark the beginning of each of the ten successive 6-sec. intervals. Abscissae numbers to the left of the arrow indicate time since $s^{R}$; those numbers to the right of the arrow indicate time since $s_{1}$ onset. emerges only after $\mathrm{S}_{1}$ 's occurrence. Similar lowering of rate and similar scalloping effects are observed for bird 268, but it would seem that this $S$ would have required shorter temporal separations between $S_{1}-S^{R}$ than we provided before its response rates would have increased in $S_{1}$ 's presence. The addition of a response contingency for $S_{1}$ 's occurrence does not appear to change essentially the findings reported earlier (Farmer \& Schoenfeld, 1966) for non-contingent introduction of $s_{1}$. This conclusion seems acceptable despite an emergent difference in effects of response contingence and non-contingence of $S_{1}$ at the longest $S_{1}-S^{R}$ interval of 60 sec. In our earlier work using the non-contingent procedure, the baseline FI pattern of responding was closely recovered at the $60 \mathrm{sec}$. separation between $S_{1}$ and the next available $S^{R}$. In the present contingent case, the low initial rates typical of FI do not emerge, and the sigmoid curvature of the function is attentuated. This difference is perhaps attributable to the fact that the addition of a response contingency for $S_{1}$ 's occurrence means an increase in the actual FI length by the amount of the average pause after $S^{R}$. This lengthened FI, in turn, acts to increase the pause after $S^{R}$ further, and this again lengthens FI even more, the reciprocating effect being one of eventually raising the mean interval between reinforcements to two or more times the programmed $60 \mathrm{sec}$. Since the first of the ten 6-sec. intervals in which data were recorded began with the first post-S ${ }^{\mathbf{R}}$ response (which also initiated the $S_{1}$ coincident with that first 6-sec. interval), the last functions plotted in Fig. 1 do not contain the pause after the preceding $\mathrm{S}^{\mathrm{R}}$; what is shown, rather, are the terminal changes in rate to $S^{R}$ that are cued off by $S_{1}$.

\section{Reference}

Farmer, J., \& Schoenfeld, W. N. Varying temporal placement of an added stimulus in a fixed-interval schedule. J.exp. Anal. Behav., $1966,9,142-148$.

\section{Note}

1. This investigation was supported by Public Health Service Research Grant MH 08006, from the National Institute of Mental Health. Reprints may be obtained from the author, Department of Psychology, Queens College of the City University of New York, Flushing, N. Y. 11367. 